\title{
Rational Selection of the 3D Structure of Biomacromolecules for Molecular Docking Studies on the Mechanism of Endocrine Disruptor Action
}

\author{
Xianhai Yang, ${ }^{\dagger}$ Huihui Liu, ${ }^{\ddagger}$ Jining Liu, ${ }^{* \dagger}{ }^{\dagger}$ Fei Li, ${ }^{\S}$ Xuehua Li, ${ }^{\|}$Lili Shi, ${ }^{\dagger}$ and Jingwen Chen ${ }^{*}, \|$ \\ ${ }^{\dagger}$ Nanjing Institute of Environmental Science, Ministry of Environmental Protection, Nanjing 210042, China \\ †jiangsu Key Laboratory of Chemical Pollution Control and Resources Reuse, School of Environmental and Biological Engineering, \\ Nanjing University of Science and Technology, Nanjing 210094, China \\ ${ }^{\S}$ Yantai Institute of Coastal Zone Research, Chinese Academy of Sciences, Yantai 264003, China \\ "Key Laboratory of Industrial Ecology and Environmental Engineering (Ministry of Education), School of Environmental Science and \\ Technology, Dalian University of Technology, Dalian 116024, China
}

ABSTRACT: Molecular modeling has become an essential tool in predicting and simulating endocrine disrupting effects of chemicals. A key prerequisite for successful application of molecular modeling lies in the correctness of $3 \mathrm{D}$ structure for biomacromolecules to be simulated. To date, there are several databases that can provide the experimentally-determined 3D structures. However, commonly, there are many challenges or disadvantageous factors, e.g., (a) lots of 3D structures for a given biomacromolecular target in the protein database; (b) the quality variability for those structures; (c) belonging to different species; (d) mutant amino acid residue in key positions, and so on. Once an inappropriate $3 \mathrm{D}$ structure of a target biomacromolecule was selected in molecular modeling, the accuracy and scientific nature of the modeling results could be inevitably affected. In this article, based on literature survey and an analysis of the 3D structure characterization of biomacromolecular targets belonging to the endocrine system in protein databases, six principles were proposed to guide the selection of the appropriate $3 \mathrm{D}$ structure of biomacromolecules. The principles include considering the species diversity, the mechanism of action, whether there are mutant amino acid residues, whether the number of protein chains is correct, the degree of structural similarity between the ligand in 3D structure and the target compounds, and other factors, e.g., the experimental $\mathrm{pH}$ conditions of the structure determined process and resolution.

\section{INTRODUCTION}

Endocrine hormones are chemical messengers secreted by endocrine glands, which play an indispensable role during the whole lifetime of an organism. For example, thyroid hormones (THs) regulate growth, differentiation, metamorphosis, and thermogenesis of vertebrates. ${ }^{1}$ A key prerequisite for endocrine hormones exerting their biological effects in humans and wildlife is that the organism maintains normal homeostasis of hormones. However, the results from epidemiological and field investigations, as well as in vivo studies, documented that many man-made chemicals, named endocrine disrupting chemicals (EDCs), could influence the homeostasis of hormones, disturb the function of the endocrine system, and consequently cause adverse health effects. ${ }^{2-4}$ According to the identified toxicity pathways, EDCs may disrupt the endocrine hormone signaling by (a) impacting the hypothalamic-pituitary-gonad/thyroid/ adrenal axis function and regulation, (b) inhibiting the hormone synthesis, (c) disrupting the hormone transport binding proteins, (d) activating/inhibiting the hormone receptor, and (e) impacting the hormone metabolism. ${ }^{1,5}$ This

\section{Rational Selection of 3D Structures of Biomacromolecules for Molecular Docking Studies}

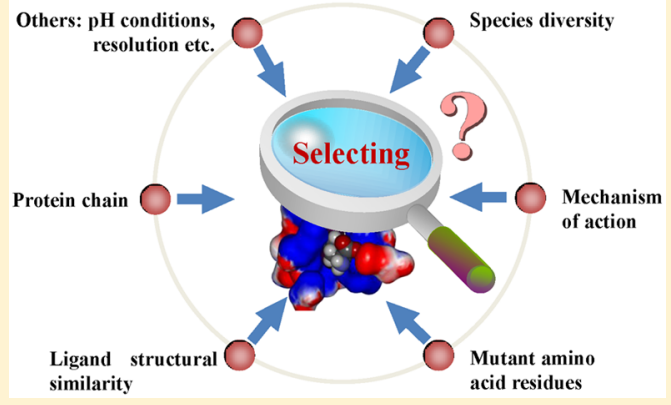

indicated that whether the endocrine hormones exert their biological effects or EDCs cause endocrine-related diseases and endocrine dysfunction, the mechanisms of endocrine disruptor action can be generalized as the interactions between small molecules and biomacromolecular targets (e.g., hormone receptor, hormone transport binding proteins, synthetase and metabolic enzymes of hormones, and so on). ${ }^{6-11}$ Thus, revealing the interaction mechanisms between the EDCs (even endocrine hormones) and biomacromolecules will pave the way for developing screening methods of EDCs and setting priority for various chemicals.

Molecular modeling adopts theoretical methods and computational technologies to investigate the interactions between a small molecule (also called ligand) and a biomacromolecule (also called receptor), on the basis of their 3D structures. Molecular modeling methods (e.g., molecular docking, molecular dynamics, hybrid quantum mechanics/

Received: July 14, 2016

Published: August 24, 2016 


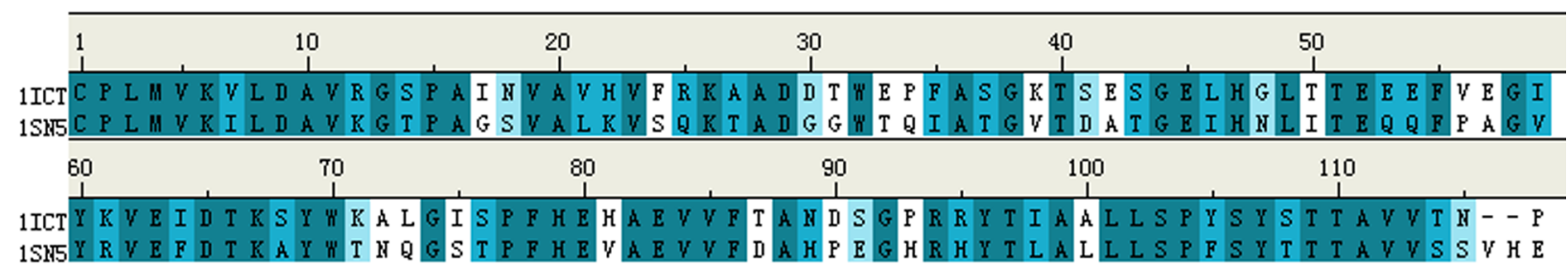

Figure 1. Amino acid sequence alignment results of transthyretins for humans (PDB ID: 1ICT) and sea breams (PDB ID: 1SN5) in one subunit.

molecular mechanics (QM/MM) methods, etc.) have become an essential tool in the study of environmental endocrine disrupting effects, ${ }^{12}$ which have been widely used to reveal the interaction mechanism between EDCs and the biomacromolecules, and to fill the data gap for EDCs on their endocrine disrupting activity, as well as set priorities. ${ }^{13-19}$

It is worth mentioning that the molecular modeling methods depend greatly on the 3D structure of biomacromolecules. Thus, a key prerequisite for the successful application of molecular modeling methods is to select an appropriate 3D structure of biomacromolecular targets. The 3D structure of a biomacromolecule could be usually obtained from various protein databases and/or predicted from corresponding amino $\mathrm{acid} /$ nucleic acid sequences. To date, various protein databases are the major sources for researchers to obtain the experimentally determined 3D structures of biomacromolecular targets. However, commonly, there are many challenges and disadvantageous factors, e.g., (a) lots of 3D structures for a given biomacromolecule in the protein database; (b) the quality variability for those structures; (c) belonging to different species; (d) mutant amino acid residues in key positions, and so on. Once the inappropriate 3D structure of the target biomacromolecule was selected in molecular modeling, the accuracy and scientific nature of the modeling results could be inevitably affected. To the best of our knowledge, there are no previous reports to address this critical issue. Therefore, the object of the present study was to propose several principles that provide guidelines for selecting appropriate macromolecules, through an in-depth literature survey and an analysis of the experimentally determined 3D structure characterization of biomacromolecules in protein databases.

\section{RESEARCH METHODS}

As stated above, the biomacromolecules in an endocrine system contained hormone receptors, hormone transport binding proteins, synthetases and metabolic enzymes of hormones, and so on. Thus, a literature review was conducted to compile the mechanisms of endocrine disruptor action and $3 \mathrm{D}$ structure characterization of the aforementioned biomacromolecules. In the present study, the endocrine biomacromolecules belonged to the hypothalamic pituitary gonadal (HPG) axis, and the hypothalamic pituitary thyroid (HPT) axis system was mainly considered. The search terms were "mechanism of EDC", "estrogen receptor (ER)", "androgen receptor (AR)", "progesterone receptor (PR)", "thyroid hormone receptor (TR)", "sex hormone binding globulin (SHBG)", "thyroid hormones transport proteins (i.e. transthyretin (TTR), thyroxine-binding globulin (TBG), and albumin (ALA))", "sulfotransferase", "hydroxysteroid dehydrogenases", and so on. In addition, the experimentally determined $3 \mathrm{D}$ structure characterization of the aforementioned biomacromolecules in protein databases was also analyzed. The $3 \mathrm{D}$ structures of the biomacromolecular target were selected from the Protein Data Bank (http://www.rcsb.org/pdb/home/home.do). On the basis of the information from the literature survey and results from the $3 \mathrm{D}$ structure characterization of the biomacromolecular targets analysis, some principles were summarized.
In order to illustrate the mentioned principles, four figures were created. All of the figures were prepared by using Discovery Studio 2.5.5 (Accelrys Software Inc.). The amino acid sequences alignment was carried out by employing the amino acid sequences from human transthyretin (PDB ID: 1ICT) and sea bream transthyretin (PDB ID: $1 \mathrm{SN} 5$ ). Figures $2-4$ were generated by using the $3 \mathrm{D}$ structure of related biomacromolecular from Protein Data Bank (http://www.rcsb. org/pdb/home/home.do).

\section{RESULTS AND DISCUSSION}

Considering the Species Diversity. The structure and function for many macromolecules seemed phylogenetically conserved across different species. For example, transthyretin (TTR) is one of the three major thyroid hormone-binding proteins in plasma and/or cerebrospinal fluid of vertebrates. Under physiological conditions, TTR mainly exists as a tetramer. ${ }^{20}$ However, the sequence identity and similarity showed considerable species variation. With TTR, the sequence identity and similarity for humans (Homo sapiens) and sea breams (Sparus aurata) were as low as $48 \%$ and $67 \%$, respectively. ${ }^{21}$

Comparing the TTR 3D structure of humans (PDB ID: 1ICT) with sea breams (PDB ID: 1SN5), it was found that there were 52 different amino acid residues in each subunit (Figure 1). Among the 52 different amino acid residues, 7 residues were located in the ligand binding domain, and the side chain of 28 amino acid residues in the sea bream TTR structure were larger than that in the human TTR structure. For example, the amino acid residues 109 and 117 in the human TTR structure were alanine and serine residues, while they were leucine and threonine residues in the sea bream structure, which may account for why the entrance of the THbinding site of sea bream transthyretin is significantly wider, while the channel is narrower. ${ }^{22}$ Those distinct amino acid residues in the 3D structure have resulted in substantial differences in the binding affinity of human TTR and sea bream TTR with EDCs, even endocrine hormone. Previous results indicated that the human TTR has higher binding affinity for 3,3',5,5'-tetraiodo-L-thyronine $\left(\mathrm{T}_{4}\right)$ than 3,3',5-triiodo-L-thyronine $\left(\mathrm{T}_{3}\right)$, whereas the reverse holds for sea bream $\mathrm{TTR}^{23}$ In addition, the binding affinity of human TTR with $\mathrm{T}_{4}$ was higher than that with all of the tested polybrominated diphenyl ethers (PBDEs) ${ }^{24}$ However, the binding potency of sea bream TTR with $\mathrm{T}_{4}$ was lower than that with the same tested PBDEs. ${ }^{25}$ Except for teleost fish, it also has been shown that TTR from amphibians, reptiles, and birds has higher affinity for $\mathrm{T}_{3}$ than for $\mathrm{T}_{4}{ }^{26}$ Therefore, during the selection of the $3 \mathrm{D}$ structure of biomacromolecules, taking the species diversity into account is necessary. Furthermore, if there is no 3D structure available for target species, we could predict the 3D structure of the target biomacromolecules by employing homology modeling or selecting the 3D structure of the target biomacromolecules from other species with high sequence identity and similarity. 
Considering the Mechanism of Action. There are two mechanisms through which EDCs exerting their effects on the hormone receptors: ${ }^{27}$ (a) agonistic effect by binding to the cellular receptor of a hormone, thereby activating normal cell response at the wrong time or to an excessive extent and (b) antagonistic effect by binding to the receptor, thereby preventing natural hormonal binding and activation of the receptor. Thus, the receptor ligands were classified into agonists and antagonists. It is well-known that hormone receptors usually undergo conformational changes after ligand binding and that different ligands are responsible for different conformations. $^{28,29}$ For example, Figure 2 illustrates the
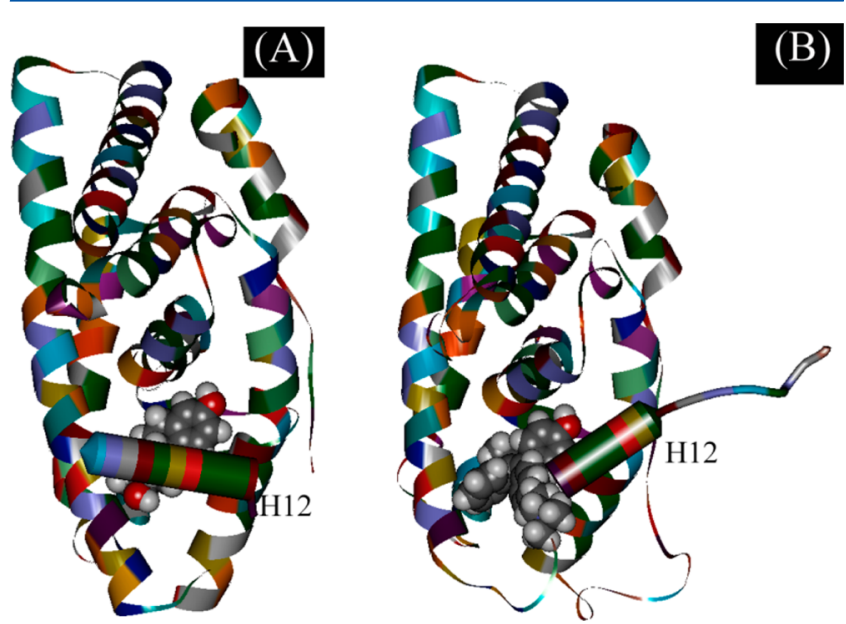

Figure 2. Position adopted by helix 12 in the human estrogen receptor with agonist (17 $\beta$-estradiol (A), PDB ID: 1ERE) and antagonist (4hydroxytamoxifen (B), PDB ID: 3ERT).

position adopted by helix 12 in the human estrogen receptor with an agonist (17 $\beta$-estradiol (A), PDB ID: 1ERE) and an antagonist (4-hydroxytamoxifen (B), PDB ID: 3ERT). As shown, the binding of antagonists (i.e., 4-hydroxytamoxifen (B)) induced a significant conformational change in the estrogen receptor ligand region through the displacement of helix 12. Except for estrogen receptor, the progesterone receptor also shares a similar phenomenon. ${ }^{30,31}$ This result indicated that the mechanism of action should be considered during selecting the $3 \mathrm{D}$ structure of biomacromolecules. It means that when EDCs exert their effects on the hormone receptors through the agonistic effect, the agonist structure should be selected; otherwise, the antagonist structure should be selected.

Considering Whether There Are Mutant Amino Acid Residues. In the protein database, some of the 3D structures contained mutant amino acid residues. If the critical amino acid residues in the ligand binding domain mutated, the results of mechanism analysis could be influenced. For example, the results from a previous study indicated that the most important amino acid residues involved in the formation of hydrogen bonding in human androgen receptor (hAR) 3D structures were Asn 705, Thr 877, Arg 752, Gln 711, and Leu 704. ${ }^{32,33}$ Under normal conditions, the no. 877 amino acid residue in hAR 3D structures was threonine. However, no. 877 amino acid residue in 1GS4 was alanine. Compared with threonine, the side chain of alanine lacks the hydroxyl group, which results in the no. 877 amino acid residue failed to form hydrogen bonds with the ligand in 1GS4. Results from Sack et al. ${ }^{34}$ indicated that the binding affinity of hAR with compounds will be changed when the no. 877 amino acid residue mutated from threonine to alanine. Recently, Sakkiah et al. ${ }^{32}$ reviewed the AR complexes in PDB. Their results indicated that the mutated no. 877 amino acid residue significantly increases AR binding affinity to estrogens and progesterone and allows AR to be activated by antiandrogens such as flutamide. Therefore, we should consider whether there are mutant amino acid residues during the selection of the 3D structure of biomacromolecules, especially to make sure whether there is a mutation of key amino acid residues in the ligand binding domain.

Considering Whether the Number of Chain Is Correct. Under normal physiological conditions, some macromolecules in the endocrine system contain several subunits. As mentioned above, the 3D structure of TTR is a homotetramer with a central hydrophobic channel, in which the two hormonebinding sites are situated between monomers a and $\mathrm{c}$ and $\mathrm{b}$ and d (Figure 3A). ${ }^{35}$ To date, only few 3D structures of human
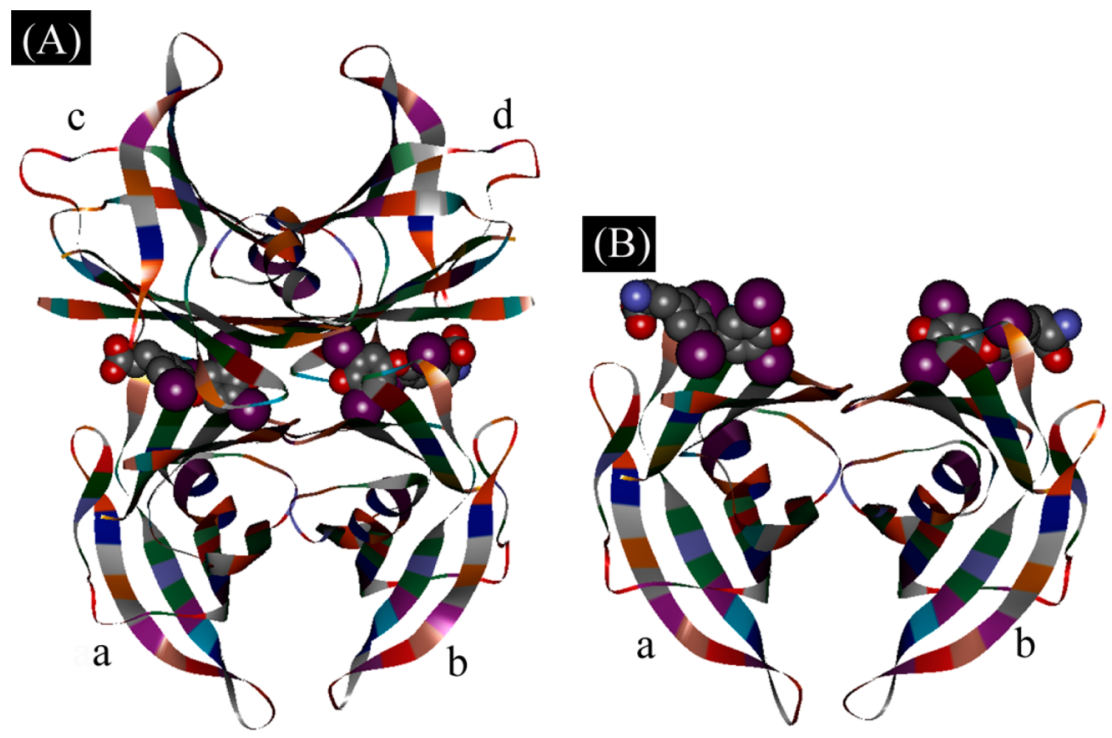

Figure 3. Normal (A, PDB ID: 1ICT) and abnormal (B, PDB ID: 2ROX) crystal structures of human transthyretin under physiological conditions. 

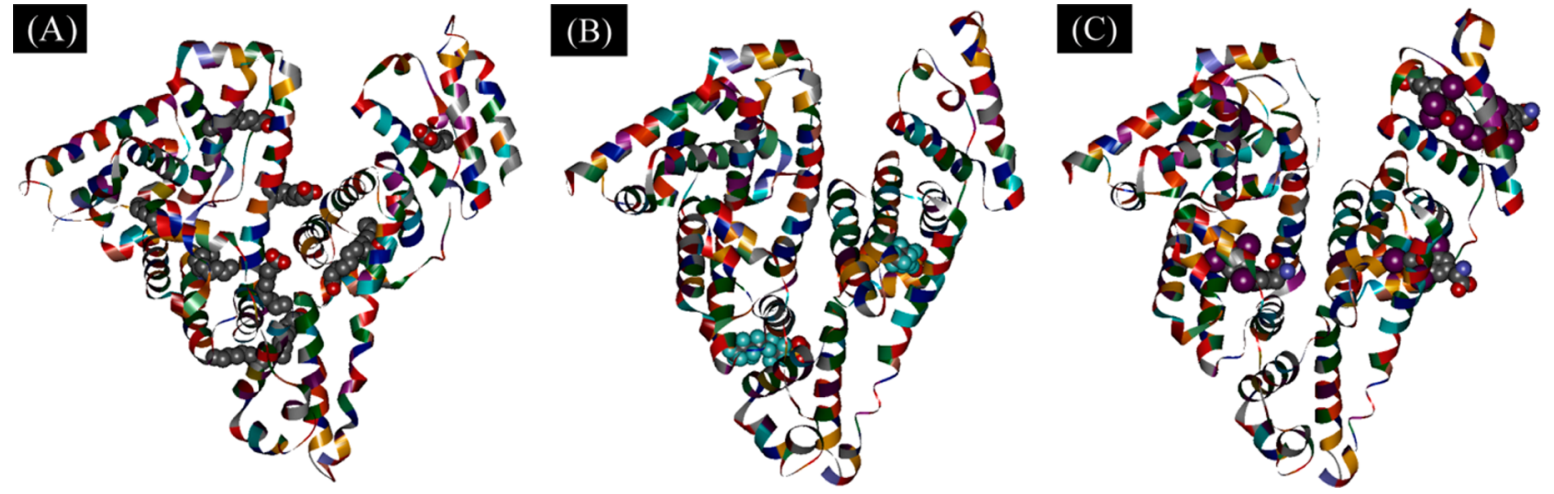

Figure 4. Binding sites of decanoic acid (A, PDB ID: 1E7E), perfluorooctanesulfonate (B, PDB ID: 4E99), and 3,3'5,5'-tetraiodo-L-thyronine (C, PDB ID: 1HK1) in serum albumin.

TTR in the PDB database contained a complete tetramer structure. The structural scheme of the incomplete 3D structure for human TTR is shown in Figure 3B. As observed in Figure $3 \mathrm{~B}$, those $3 \mathrm{D}$ structures also contained two monomers. However, the ligand binding domain is incomplete. If those inappropriate 3D structures of human TTR were selected and used to perform molecular modeling, the inappropriate ligand binding site may be defined, which will inevitably affect the accuracy and scientific nature of the modeling results. Therefore, before selecting the 3D structure, researchers need to fully understand the amount of the peptide chain under physiological conditions for the target biomolecules.

Considering the Degree of Structural Similarity between the Target Compounds and the Ligand in the 3D Structure. As is well-known, molecules with similar structural features are thought to exhibit and elicit parallel physicochemical properties, environmental behavior, and toxicological effects. Similarly, molecules with similar structural features also are expected to share similar modes of action and binding sites during the interaction between ligands and biomacromolecules. In this case, the structural similarity between the ligand in the $3 \mathrm{D}$ structure and the target compounds should be considered, especially for the biomacromolecules containing several ligand binding sites. For example, the albumin contained several ligand binding sites (Figure 4). Analysis results of the albumin 3D structure indicated that there are 9 binding sites for long-chain fatty acids, $^{36} 2$ for perfluorooctanesulfonate, ${ }^{37}$ and 4 for thyroxine. ${ }^{38}$ Thus, the structural similarity between the ligand in the $3 \mathrm{D}$ structure and the target compounds should not be neglected when selecting the $3 \mathrm{D}$ structure.

Considering Other Factors, for Example, the Experimental $\mathrm{pH}$ Conditions of the Structure Determined Process and Resolution. For many biomacromolecules, their molecular structures contain ionizable alkaline or acidic residues. ${ }^{39}$ Those residues can ionize depending on the $\mathrm{pH}$ of the environment and their $\mathrm{p} K_{\mathrm{a}}$ values, which means that the same alkaline or acidic residue in a given biomacromolecule may have distinct protonation or deprotonation states under different $\mathrm{pH}$ conditions. The protonated or deprotonated residues could form ionic/electrostatic interactions with other residues or ligands in biomacromolecules. It could be speculated that the aforesaid ionic/electrostatic interactions may be $\mathrm{pH}$ dependent. It is well-known that the structural stability and function of many biomacromolecules are governed by ionic/electrostatic interactions between protonated or deprotonated residues and other residues/ligand. ${ }^{40,41}$ Thus, the quaternary and tertiary structure may also show a distinct $\mathrm{pH}$-dependent conformational characteristic. For example, Palaninathan et al. $^{42}$ investigated the effect of acidification on the quaternary and tertiary structures of TTR. Indeed, it was found that the conformation of the $3 \mathrm{D}$ structure could be influenced by the $\mathrm{pH}$ conditions of crystallization process. Compared with the wild-type TTR structure at $\mathrm{pH} 7.4$, the EF helix (residues 75-81)-loop (residues 82-90) region, in the structures was influenced at $\mathrm{pH} 3.5$ and 4.0. In the 3D structure at $\mathrm{pH} 4.0$, the acidic residues within the $\mathrm{EF}$ helix-loop region undergo significant conformational changes, which instigate a movement of the EF helix-loop region and make the residues Lys 70, Lys 76, His 88, and His 90 orient their side chains toward these acidic residues. In the structure at $\mathrm{pH} 3.5$, the EF helix-loop region is completely disordered. Those results indicate that the crystallization process is $\mathrm{pH}$-dependent, which means that the $\mathrm{pH}$ conditions for crystallizing target biomacromolecules should be identical to the normal physiological $\mathrm{pH}$ conditions or experimental $\mathrm{pH}$ conditions.

After considering the aforementioned factors, more than one $3 \mathrm{D}$ structure could be selected. In this case, the resolution of the $3 \mathrm{D}$ structure can be further used to determine which one was better. Usually, preference should be given to the $3 \mathrm{D}$ structure with good resolution.

\section{CONCLUSIONS}

The interaction between xenobiotics and biomacromolecules in the endocrine system was the key molecular initiating event of the endocrine-related harmful effects. ${ }^{43,44}$ Deciphering the mechanism between xenobiotics and biomacromolecules will be conducive to develop the endocrine-based adverse outcome pathway. ${ }^{45-47}$ Molecular modeling methods were widely used to reveal the interaction mechanism. Obtaining an appropriate 3-D structure of biomacromolecular targets was the key prerequisite for the successful application of molecular modeling methods. The six principles presented in this study could help us in evaluating whether the selected biomacromolecules from the protein databases is appropriate or not. Thus, the principles are timely and important, and meet the present needs of molecular modeling and improve our understanding of the endocrine disrupting effect. In addition, the homology modeling constructs an atomic-resolution model of the "target" biomacromolecule based on its amino acid/nucleic acid sequence and an experimental 3D structure of a related homologous biomacromolecule (the "template"). The princi- 
ples also could assist us to evaluate the reasonability of the selected "template" and predicted biomacromolecules.

\section{AUTHOR INFORMATION}

\section{Corresponding Authors}

*(J.L.) Nanjing Institute of Environmental Sciences, Ministry of Environmental Protection, No.8 Jiangwangmiao Steet, Nanjing 210042, China. Tel: +86-25-85287608. Fax: +86-2585287611. E-mail: ljn@nies.org.

*(J.C.) School of Environmental Science and Technology, Dalian University of Technology, Linggong Road 2, Dalian 116024, China. Tel/Fax: 86-411-8470 6269. E-mail: jwchen@ dlut.edu.cn.

\section{Funding}

This work was supported by the National Natural Science Foundation of China (Nos. 21507038 and 21507061) and Natural Science Foundation of Jiangsu Province (Nos. BK20150771 and BK20151100).

\section{Notes}

The authors declare no competing financial interest.

\section{ABBREVIATIONS}

EDCs, endocrine disrupting chemicals; hAR, human androgen receptor; PBDEs, polybrominated diphenyl ethers; QM/MM, hybrid quantum mechanics/molecular mechanics method; $\mathrm{T}_{3}$, 3,3',5-triiodo-L-thyronine; $\mathrm{T}_{4}, 3,3^{\prime}, 5,5^{\prime}$-tetraiodo-L-thyronine; THs, thyroid hormones; TTR, transthyretin

\section{REFERENCES}

(1) Li, F., Yang, X. H., Chen, J. W., and Lyakurwa, F. (2015) QSARs on the Thyroid Hormone Effects of Polybrominated Diphenyl Ether (PBDE) Derivatives, in Persistent Organic Pollutants (POPs): Analytical Techniques, Environmental Fate and Biological Effects (Zeng, E. Y., Eds.) pp 547-586, Comprehensive Analytical Chemistry, 67, Elsevier, The Netherlands.

(2) Diamanti-Kandarakis, E., Bourguignon, J. P., Giudice, L. C., Hauser, R., Prins, G. S., Soto, A. M., Zoeller, R. T., and Gore, A. C. (2009) Endocrine-disrupting chemicals: an Endocrine Society scientific statement. Endocr. Rev. 30, 293-342.

(3) United Nations Environment Programme/World Health Organization (UNEP/WHO) (2013) State of the Science of Endocrine Disrupting Chemicals-2012 (Bergman, A., Heindel, J. J., Jobling, S., Kidd, K. A., and Zoeller, R. T., Eds.), UNEP/WHO, Geneva, Switzerland.

(4) Gore, A. C., Chappell, V. A., Fenton, S. E., Flaws, J. A., Nadal, A., Prins, G. S., Toppari, J., and Zoeller, R. T. (2015) EDC-2: The Endocrine Society's Second Scientific Statement on EndocrineDisrupting Chemicals. Endocr. Rev. 36, E1-E150.

(5) Liu, H. H., Yang, X. H., and Lu, R. (2016) Development of classification model and QSAR model for predicting binding affinity of endocrine disrupting chemicals to human sex hormone-binding globulin. Chemosphere 156, 1-7.

(6) Greim, H. (2005) Chemicals with endocrine-disrupting potential: a threat to human health? Angew. Angew. Chem., Int. Ed. 44, 55685574.

(7) Connolly, L., Ropstad, E., and Verhaegen, S. (2011) In vitro bioassays for the study of endocrine-disrupting food additives and contaminants. TrAC, Trends Anal. Chem. 30, 227-238.

(8) Miller, M. D., Crofton, K. M., Rice, D. C., and Zoeller, R. T. (2009) Thyroid-disrupting chemicals: interpreting upstream biomarkers of adverse outcomes. Environ. Health Perspect. 117, 10331041.

(9) Bushnell, P. J., Kavlock, R. J., Crofton, K. M., Weiss, B., and Rice, D. C. (2010) Behavioral toxicology in the 21st century: challenges and opportunities for behavioral scientists. Summary of a symposium presented at the annual meeting of the neurobehavioral teratology society, June, 2009. Neurotoxicol. Teratol. 32, 313-328.

(10) Murk, A. J., Rijntjes, E., Blaauboer, B. J., Clewell, R., Crofton, K. M., Dingemans, M. M., Furlow, J. D., Kavlock, R., Köhrle, J., Opitz, R, Traas, T., Visser, T. J., Xia, M., and Gutleb, A. C. (2013) Mechanismbased testing strategy using in vitro approaches for identification of thyroid hormone disrupting chemicals. Toxicol. In Vitro 27, 13201346.

(11) Maqbool, F., Mostafalou, S., Bahadar, H., and Abdollahi, M. (2016) Review of endocrine disorders associated with environmental toxicants and possible involved mechanisms. Life Sci. 145, 265-273.

(12) Rabinowitz, J. R., Goldsmith, M. R., Little, S. B., and Pasquinelli, M. A. (2008) Computational molecular modeling for evaluating the toxicity of environmental chemicals: prioritizing bioassay requirements. Environ. Health Perspect. 116, 573-577.

(13) Rabinowitz, J. R., Little, S. B., Laws, S. C., and Goldsmith, M. R. (2009) Molecular modeling for screening environmental chemicals for estrogenicity: use of the toxicant-target approach. Chem. Res. Toxicol. 22, 1594-1602.

(14) Li, F., Xie, Q., Li, X. H., Li, N., Chi, P., Chen, J. W., Wang, Z. J., and Hao, C. (2010) Hormone activity of hydroxylated polybrominated diphenyl ethers on human thyroid receptor-beta: in vitro and in silico investigations. Environ. Health Perspect. 118, 602-606.

(15) Yang, X. H., Xie, H. B., Chen, J. W., and Li, X. H. (2013) Anionic phenolic compounds bind stronger with transthyretin than their neutral forms: nonnegligible mechanisms in virtual screening of endocrine disrupting chemicals. Chem. Res. Toxicol. 26, 1340-1347.

(16) Zhuang, S. L., Zhang, C. L., and Liu, W. P. (2014) Atomic insights into distinct hormonal activities of Bisphenol A analogues toward PPAR $\gamma$ and ER $\alpha$ receptors. Chem. Res. Toxicol. 27, 1769-1779.

(17) Ng, H. W., Shu, M., Luo, H., Ye, H., Ge, W. G., Perkins, R., Tong, W. D., and Hong, H. X. (2015) Estrogenic activity data extraction and in silico prediction show the endocrine disruption potential of bisphenol A replacement compounds. Chem. Res. Toxicol. $28,1784-1795$

(18) Kolšek, K., Mavri, J., Dolenc, M. S., Gobec, S., and Turk, S. (2014) Endocrine Disruptome-An Open Source Prediction Tool for Assessing Endocrine Disruption Potential through Nuclear Receptor Binding. J. Chem. Inf. Model. 54, 1254-1267.

(19) Plošnik, A., Vračko, M., and Mavri, J. (2015) Computational study of binding affinity to nuclear receptors for some cosmetic ingredients. Chemosphere 135, 325-334.

(20) Prapunpoj, P., and Leelawatwattana, L. (2009) Evolutionary changes to transthyretin: structure-function relationships. FEBS J. 276 , $5330-5341$

(21) Power, D. M., Elias, N. P., Richardson, S. J., Mendes, J., Soares, C. M., and Santos, C. R. (2000) Evolution of the thyroid hormonebinding protein, transthyretin. Gen. Comp. Endocrinol. 119, 241-255.

(22) Eneqvist, T., Lundberg, E., Karlsson, A., Huang, S., Santos, C. R., Power, D. M., and Sauer-Eriksson, A. E. (2004) High resolution crystal structures of piscine transthyretin reveal different binding modes for triiodothyronine and thyroxine. J. Biol. Chem. 279, 2641126416.

(23) Richardson, S. J. (2009) Evolutionary changes to transthyretin: evolution of transthyretin biosynthesis. FEBS J. 276, 5342-5356.

(24) Hamers, T., Kamstra, J. H., Sonneveld, E., Murk, A. J., Kester, M. H., Andersson, P. L., Legler, J., and Brouwer, A. (2006) In vitro profiling of the endocrine-disrupting potency of brominated flame retardants. Toxicol. Sci. 92, 157-173.

(25) Morgado, I., Hamers, T., Van der Ven, L., and Power, D. M. (2007) Disruption of thyroid hormone binding to sea bream recombinant transthyretin by ioxinyl and polybrominated diphenyl ethers. Chemosphere 69, 155-163.

(26) Ucán-Marín, F., Arukwe, A., Mortensen, A., Gabrielsen, G. W., Fox, G. A., and Letcher, R. J. (2009) Recombinant transthyretin purification and competitive binding with organohalogen compounds in two gull species (Larus argentatus and Larus hyperboreus). Toxicol. Sci. 107, 440-450. 
(27) Shanle, E. K., and Xu, W. (2011) Endocrine disrupting chemicals targeting estrogen receptor signaling: identification and mechanisms of action. Chem. Res. Toxicol. 24, 6-19.

(28) Brzozowski, A. M., Pike, A. C., Dauter, Z., Hubbard, R. E., Bonn, T., Engström, O., Ohman, L., Greene, G. L., Gustafsson, J. A., and Carlquist, M. (1997) Molecular basis of agonism and antagonism in the oestrogen receptor. Nature 389, 753-758.

(29) Celik, L., Davey, J., Lund, D., and Schiøtt, B. (2008) Exploring interactions of endocrine-disrupting compounds with different conformations of the human estrogen receptor alpha ligand binding domain: a molecular docking study. Chem. Res. Toxicol. 21, 21952206.

(30) Williams, S. P., and Sigler, P. B. (1998) Atomic structure of progesterone complexed with its receptor. Nature 393, 392-396.

(31) Madauss, K. P., Grygielko, E. T., Deng, S. J., Sulpizio, A. C., Stanley, T. B., Wu, C., Short, S. A., Thompson, S. K., Stewart, E. L., Laping, N. J., Williams, S. P., and Bray, J. D. (2007) A structural and in vitro characterization of asoprisnil: a selective progesterone receptor modulator. Mol. Endocrinol. 21, 1066-1081.

(32) Sakkiah, S., Ng, H. W., Tong, W. D., and Hong, H. X. (2016) Structures of androgen receptor bound with ligands: advancing understanding of biological functions and drug discovery. Expert Opin. Ther. Targets, DOI: 10.1080/14728222.2016.1192131.

(33) Yang, X. H., Liu, H. H., Yang, Q., Liu, J. N., Chen, J. W., and Shi, L. L. (2016) Predicting anti-androgenic activity of bisphenols using molecular docking and quantitative structure-activity relationships. Chemosphere 163, 373-381.

(34) Sack, J. S., Kish, K. F., Wang, C., Attar, R. M., Kiefer, S. E., An, Y., Wu, G. Y., Scheffler, J. E., Salvati, M. E., Krystek, S. R., Jr., Weinmann, R, and Einspahr, H. M. (2001) Crystallographic structures of the ligand-binding domains of the androgen receptor and its T877A mutant complexed with the natural agonist dihydrotestosterone. Proc. Natl. Acad. Sci. U. S. A. 98, 4904-4909.

(35) Wojtczak, A., Cody, V., Luft, J. R., and Pangborn, W. (1996) Structures of human transthyretin complexed with thyroxine at $2.0 \mathrm{~A}$ resolution and $3^{\prime}, 5^{\prime}$-dinitro-N-acetyl-L-thyronine at $2.2 \mathrm{~A}$ resolution. Acta Crystallogr., Sect. D: Biol. Crystallogr. 52, 758-765.

(36) Bhattacharya, A. A., Grüne, T., and Curry, S. (2000) Crystallographic analysis reveals common modes of binding of medium and long-chain fatty acids to human serum albumin. J. Mol. Biol. 303, 721-732.

(37) Luo, Z., Shi, X., Hu, Q., Zhao, B., and Huang, M. (2012) Structural evidence of perfluorooctane sulfonate transport by human serum albumin. Chem. Res. Toxicol. 25, 990-992.

(38) Petitpas, I., Petersen, C. E., Ha, C. E., Bhattacharya, A. A., Zunszain, P. A., Ghuman, J., Bhagavan, N. V., and Curry, S. (2003) Structural basis of albumin-thyroxine interactions and familial dysalbuminemic hyperthyroxinemia. Proc. Natl. Acad. Sci. U. S. A. 100, 6440-6445.

(39) Russo Krauss, I., Merlino, A., Vergara, A., and Sica, F. (2013) An overview of biological macromolecule crystallization. Int. J. Mol. Sci. 14, 11643-11691.

(40) Lee, K. K., Fitch, C. A., and García-Moreno, E. B. (2002) Distance dependence and salt sensitivity of pairwise, coulombic interactions in a protein. Protein Sci. 11, 1004-1016.

(41) Kumar, S., and Nussinov, R. (2002) Relationship between ion pair geometries and electrostatic strengths in proteins. Biophys. J. 83, $1595-1612$.

(42) Palaninathan, S. K., Mohamedmohaideen, N. N., Snee, W. C., Kelly, J. W., and Sacchettini, J. C. (2008) Structural insight into pHinduced conformational changes within the native human transthyretin tetramer. J. Mol. Biol. 382, 1157-1167.

(43) Allen, T. E., Goodman, J. M., Gutsell, S., and Russell, P. J. (2014) Defining molecular initiating events in the adverse outcome pathway framework for risk assessment. Chem. Res. Toxicol. 27, 21002112.

(44) Dent, M. P., Carmichael, P. L., Jones, K. C., and Martin, F. L. (2015) Towards a non-animal risk assessment for anti-androgenic effects in humans. Environ. Int. 83, 94-106.
(45) Ankley, G. T., Bennett, R. S., Erickson, R. J., Hoff, D. J., Hornung, M. W., Johnson, R. D., Mount, D. R., Nichols, J. W., Russom, C. L., Schmieder, P. K., Serrrano, J. A., Tietge, J. E., and Villeneuve, D. L. (2010) Adverse outcome pathways: a conceptual framework to support ecotoxicology research and risk assessment. Environ. Toxicol. Chem. 29, 730-741.

(46) Vinken, M. (2013) The adverse outcome pathway concept: a pragmatic tool in toxicology. Toxicology 312, 158-165.

(47) Villeneuve, D. L., Crump, D., Garcia-Reyero, N., Hecker, M., Hutchinson, T. H., LaLone, C. A., Landesmann, B., Lettieri, T., Munn, S., Nepelska, M., Ottinger, M. A., Vergauwen, L., and Whelan, M. (2014) Adverse outcome pathway (AOP) development I: strategies and principles. Toxicol. Sci. 142, 312-320. 